\title{
Mechanisms underlying mycobacterial infections
}

\author{
Helena I. Boshoff ${ }^{1}$ and Kapil Tahlan ${ }^{2}$ \\ ${ }^{1}$ Tuberculosis Research Section, National Institute of Allergy and Infectious Diseases, National \\ Institutes of Health, Bethesda, U.S.A \\ ${ }^{2}$ Department of Biology, Memorial University of Newfoundland, Canada
}

\begin{abstract}
This issue of Drug Discovery Today summarizes the key aspects of mycobacterial biology that are central to the ability of Mycobacterium tuberculosis (MTb) to infect, grow, survive and transmit between its human hosts. This issue may seem particularly topical as we reach the halfway point of the Global Plan to Stop TB 2006-2015 launched by the Stop TB Partnership. In this plan the goal is to have started to reverse the incidence of tuberculosis (TB) and have $100 \%$ access to TB chemotherapy by 2015. Success of this global partnership will allow 14 million lives to be saved and will significantly improve the economies of those countries that bear the burden of this debilitating disease. Key to the success of this plan will be the introduction of new drugs as well as new vaccines. Although there are already several drugs and vaccines in the pre-clinical and clinical testing stages of the drug/vaccine development pipeline, new candidates are still urgently required to increase the probability of making a significant impact on global TB incidence. Particularly pertinent are the 2 billion people who are estimated to be latently infected with MTb which pose a future reservoir of this pathogen. Treatment of latent TB will require development of novel strategies to eradicate the bacilli that characterize the chemotherapeutically recalcitrant form of this disease. Development of better strategies to control TB require insight into the transmission chains of the pathogen since, as discussed by $\mathrm{Gao}$ and $\mathrm{Li}$, the transmission of drug resistant strains of MTb have previously been grossly underestimated. The management of highly drug resistant TB is critical in preventing global dissemination of such strains and this review highlights the controversial politics of case management. Central to development of vaccines and drugs is a better understanding of the pathogen in the context of the host environment. Reviews included in this issue deal with some of the most pertinent topics that have molded current hypotheses of the critical mechanisms that explain MTb pathogenesis.
\end{abstract}

The spectrum of disease caused by MTb ranges from initial infection controlled by innate or acquired immune mechanisms to disease with no associated clinical symptoms due to the control of the pathogen by host immunity, to various ranges of clinical disease severity in pulmonary or disseminated TB. The review by Coscolla and Gagneux provide a comprehensive overview of the use of genomic sequence data in predicting and analyzing MTb pathogenesis and infectivity. This review highlights underlying reasons for discrepancies between studies aimed at associating strain genotypes with disease phenotypes such as transmission, progression to active disease, extra-pulmonary dissemination, relapse, drug resistance and disease severity. Their sobering conclusion is that the data accumulated from all these studies have only hinted at the association of genotype with disease diversity and that more data is needed. The authors conclude by stressing the importance of integrating host, pathogen,

\footnotetext{
Publisher's Disclaimer: This is a PDF file of an unedited manuscript that has been accepted for publication. As a service to our customers we are providing this early version of the manuscript. The manuscript will undergo copyediting, typesetting, and review of the resulting proof before it is published in its final citable form. Please note that during the production process errors may be discovered which could affect the content, and all legal disclaimers that apply to the journal pertain.
} 
geographical and other environmental data in "systems epidemiological approaches" to derive meaningful conclusions about the association of pathogen genotype with host and disease.

The evolution of MTb strains driven by host immunity and aspects of human genotypic diversity relies on bacterial DNA repair pathways. These pathways further drive the generation of drug resistance mutations and associated compensatory mutations that ameliorate any fitness costs associated with mutation in the essential functions encoded by current drug targets. DNA repair mechanisms are also critical for surviving the onslaught on host immune defenses in addition to DNA damage sustained by endogenous reactive molecules produced by various metabolic processes. Dr. Warner provides insight into the field of mycobacterial DNA repair and how these mechanisms often diverge from other bacterial systems for balancing chromosome maintenance and integrity with genomic evolution in the face of environmental challenges.

Astarie-Dequeker et al. review the role of mycobacterial lipids in macrophage invasion, the subsequent phagosomal arrest and alternations in antigen presentation that are thought to be critical for MTb pathogenesis. They discuss the subtle interplay of pro-inflammatory as opposed to anti-inflammatory immune responses generated by unique mycobacterial lipids. This review also includes previously unpublished data that suggest the role of phenoglycolipid-1 in host cell invasion and of phthiocerol dimycocerosate in blocking phagosome maturation. It is clear that the study of mycobacterial lipids will remain a fruitful field of study for many years to come, and will lead to new insight in to how this highly successful group of pathogens modulate and evade the immune system to promote growth, long-term survival, dissemination and transmission within and between human hosts. Despite the importance of mycobacterial lipids in host pathogenesis, the MTb cell wall has long been thought to be a poor target for drugs developed with the aim of targeting latent disease. The review by Kana and Mizrahi raise important questions that challenge this hypothesis. In this review, the putative role of the $5 \mathrm{MTb}$ resuscitation promoting factors in peptidoglycan degradation is discussed and how bioactive muropeptides may play a role not only in signaling to the host, but also in peptidoglycan turnover in non-replicating organisms or in MTb during reactivation from a state of metabolic arrest. This raises prospects for development of these or their associated enzymes as targets for drug and vaccine development for latent tuberculosis.

Targeting virulence mechanisms of pathogens as a means to attenuate the organism, making it an "easier" target for clearance by the immune system, has been a topic of considerable debate. This approach has never been attempted for TB despite the generally incomplete success of drugs that target single essential targets to eliminate MTb from host tissues. Of the 5 type VII secretion systems encoded by MTb, two have been associated with aspects of pathogenesis with one of these, ESX-1, being a major virulence factor of this pathogen. Chen et al. review potential strategies of targeting the ESX-1 secretion system for anti-tubercular drug development, discussing strategies that could potentially employ small molecules to target ATPases, serine proteases and certain protein-protein interactions that are required for ESX-1 function, in addition to targeting the transcription of ESX-1 components directly and indirectly. Included in their list of potential targets for development of anti-virulence drugs, are the ESX-1 substrates that interact with host components to modulate cytokine production, host cell lysis, granuloma formation, phagosome maturation arrest and apoptosis. Future work on the function of the type VII secretion system substrates will likely generate a wealth of information about the applicability of such systems and their substrates as drug targets at various stages of TB disease.

Dr. Waddell reviews the transcriptional results that have been reported of MTb growth during in vivo-relevant in vitro growth conditions and in host cells, and puts these in context of bacterial metabolism under these conditions. The results from various in vivo studies, whether in animals 
ranging from mice to primates, or human patients, have in some cases conflicted with accepted hypotheses about mycobacterial metabolism. This review summarizes these findings and provides an insight into how MTb metabolism adapts to changing environmental signals at various stages of disease. Understanding the relative roles of various branches of metabolism in response to host microenvironments may point to potential targets for drug development or at the very least explain the failure of some enzyme inhibitors in clearing mycobacteria in vivo.

Small non coding RNAs (sRNAs) have been identified in all branches of life and have received considerable attention in many pathogenic bacteria. On the other hand, the identification of sRNAs in mycobacteria and their function has received little attention until recently, where approximately 20 sRNAs were identified in MTb. The review by Arnvig and Young is probably the first of its kind to be published on the subject since not much is currently known about the field. The authors describe how sRNAs regulate gene expression in other pathogenic bacteria in response to temperature, acid, oxidative and nutrient stress, in addition to regulating iron homeostasis. They also describe some of the properties of mycobacterial sRNAs and discuss some of their own work currently being pursued. This emerging field will likely revise many paradigms about our understanding of mycobacterial adaptation to its host environment and how this relates to disease.

This issue closes with a review that stresses the importance of integrating data from a multitude of platforms through systems biology approaches. Such platforms do not only include data collected from high-throughput approaches such as transcriptomics, proteomics, lipidomics, metabolomics, protein-nucleic and protein-protein interaction networks, but also data accrued from studies of individual metabolic processes to studies of the pathogen in human TB patients by assimilating data from clinical, imaging, host immunologic response, bacterial genome as well as human genotype studies.

Collectively these reviews provide insight into some of the most relevant aspects of the bacterial mechanisms that underlie TB infections in human populations. Some of the questions raised in these reviews may lead to the formulation of testable hypotheses about those mechanisms that shape disease progression, transmission and the development of drug resistance. In turn, this information will be crucial in developing strategies to ultimately defeat this pathogen.

\section{Acknowledgments}

This work was supported in part by the Intramural Research Program of the NIH, NIAID.

Drug Discov Today Dis Mech. Author manuscript; available in PMC 2011 April 1. 


\section{Biographies}

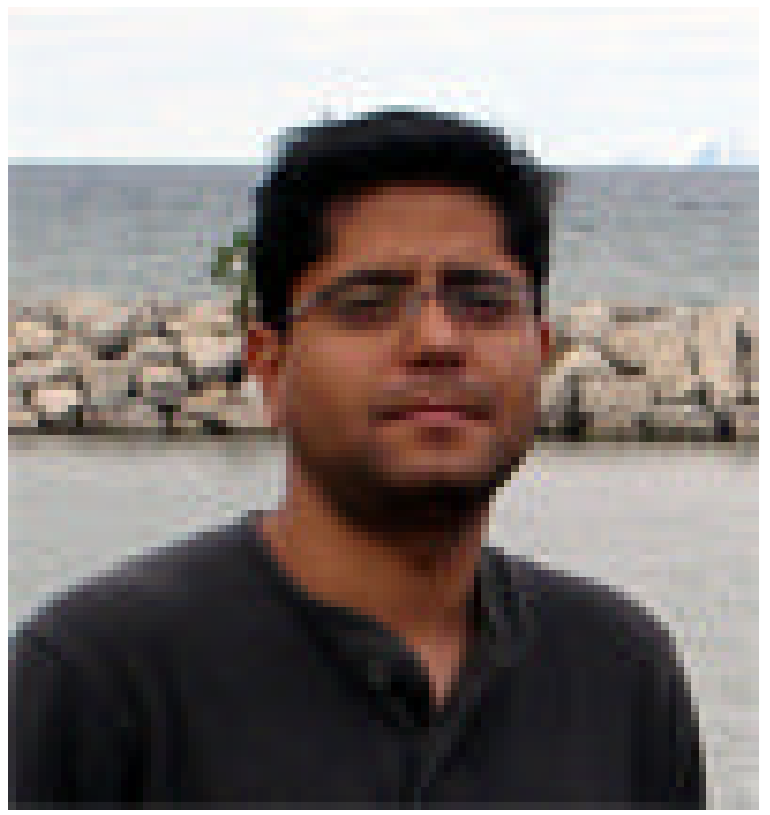

Helena I. Boshoff is a staff scientist in the Tuberculosis Research Section of the National Institute of Allergy and Infectious Diseases at the National Institutes of Health. She obtained her Ph.D. from the University of the Witwatersrand (South Africa). The Tuberculosis Research Section focuses on development of strategies for improving the chemotherapy of tuberculosis through a multitude of projects ranging from clinical studies to drug development work to basic biology research to understand the metabolism of Mycobacterium tuberculosis under diseaserelevant conditions. She serves on the editorial advisory board of the journal "Tuberculosis".

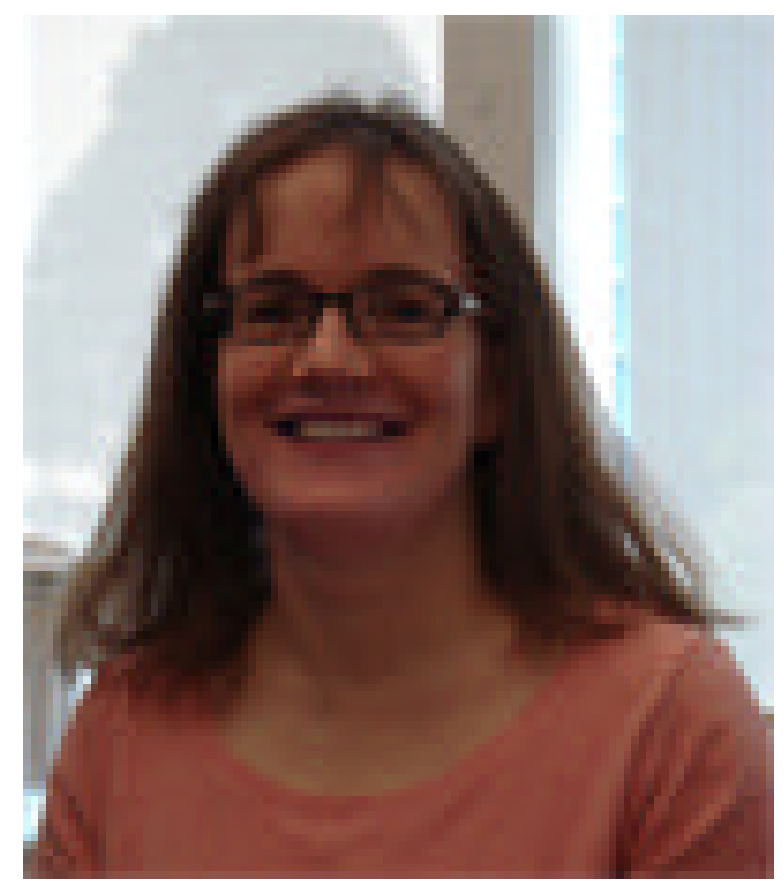


Kapil Tahlan is an assistant professor at the Memorial University of Newfoundland in Canada. He was previously a visiting fellow at the Tuberculosis Research Section of the National Institute of Allergy and Infectious Diseases at the United States National Institutes of Health and a postdoctoral fellow at McMaster University in Canada. He obtained his Ph.D. from the University of Alberta in Canada and is currently interested in the field of antibiotic discovery/ resistance and prokaryotic transcriptional regulatory mechanisms. 